\title{
Surrogate Approaches for Neutron Capture
}

J. E. Escher, F. S. Dietrich, N. D. Scielzo

May 27, 2010

International Conference on Nuclear Data for Science and Technology Jeju Island, South Korea April 26, 2010 through April 30, 2010 
This document was prepared as an account of work sponsored by an agency of the United States government. Neither the United States government nor Lawrence Livermore National Security, LLC, nor any of their employees makes any warranty, expressed or implied, or assumes any legal liability or responsibility for the accuracy, completeness, or usefulness of any information, apparatus, product, or process disclosed, or represents that its use would not infringe privately owned rights. Reference herein to any specific commercial product, process, or service by trade name, trademark, manufacturer, or otherwise does not necessarily constitute or imply its endorsement, recommendation, or favoring by the United States government or Lawrence Livermore National Security, LLC. The views and opinions of authors expressed herein do not necessarily state or reflect those of the United States government or Lawrence Livermore National Security, LLC, and shall not be used for advertising or product endorsement purposes. 


\title{
SURROGATE APPROACHES FOR NEUTRON CAPTURE
}

\author{
J.E. ESCHER* , F.S. DIETRICH, and N.D. SCIELZO \\ Lawrence Livermore National Laboratory, Livermore, CA, USA \\ ${ }^{*}$ Corresponding author. E-mail : escher1@1lnl.gov \\ Received \\ Accepted for Publication
}

The prospects for obtaining neutron capture cross sections indirectly from surrogate measurements are discussed. The surrogate reactions approach has been successfully employed to determine (n,f) cross sections from observed fission decays of compound nuclei created with the help of light-ion inelastic scattering or transfer reactions. The challenges encountered in applications of the method to capture reactions are considered. Case studies are presented that shed light on the accuracy to be expected from this approach, and ideas for improving the resulting cross sections are discussed.

KEYWORDS : Surrogate reactions, neutron capture, s process, fission

\section{THE NEED FOR INDIRECT APPROACHES}

Many areas of basic and applied nuclear physics require cross sections for neutron-capture reactions that proceed through a compound nucleus. Capture reactions occurring in the astrophysical $\mathrm{s}$ and $\mathrm{r}$ processes produce the majority of the heavy elements beyond iron, and their cross sections are necessary input for models of these processes and of stellar evolution. The abundance patterns predicted by these models can, sometimes very sensitively, depend on specific cross sections. Cross sections for neutron capture by s-process branch-point nuclei, for example, are needed to properly interpret measured abundances and to draw conclusions about stellar conditions, such as temperature or neutron fluxes, at the time of the capture. Capture cross sections also provide constraints on models of the $r$ process, as r-process abundances are typically determined by subtracting calculated s-process abundances from measured total abundances. Despite improved experimental techniques for measuring reaction rates involving short-lived targets, not all cross sections of interest have been determined. Challenging cases, for which no direct measurements exist, include low-energy $\left(E_{n} \approx 1-200 \mathrm{keV}\right)$ neutron capture on ${ }^{95} \mathrm{Zr}$ and ${ }^{153} \mathrm{Gd}$, with half lives of $\mathrm{t}_{1 / 2}=64$ and 242 days, respectively. Determining capture cross sections is also important for exploiting nuclear energy in a clean and safe manner: To understand the options for reducing nuclear waste through transmutation, to design advanced nuclear power reactors that employ the closed fuel cycle, and for exploring alternative fuel cycles, many capture and fission cross sections have to be known. Not all required cross sections can be measured directly in the near future. And while there have been important advances in understanding and reducing the uncertainties of calculations, data from indirect measurements can provide useful constraints on the calculations and increase confidence in evaluations.
This paper explores the prospects for obtaining cross sections of compound-nuclear reactions, in particular neutron-capture reactions, indirectly via the surrogate reaction method. In the next section, the surrogate formalism is summarized and applications to $(\mathrm{n}, \mathrm{f})$ reactions are briefly considered. Challenges that particularly affect capture measurements are discussed in Sec. 3. and case studies for (n, $\gamma)$ reactions in various areas of the nuclear chart are presented in Sec. 4. In Sec. 5., possibilities for improving current methods are considered; closing remarks follow in Sec. 6.

\section{THE SURROGATE METHOD}

The surrogate nuclear reaction technique combines experiment with theory to obtain cross sections for compoundnuclear $(\mathrm{CN})$ reactions, $a+A \rightarrow B^{*} \rightarrow c+C$, involving targets $(A)$ that are difficult or impossible to obtain $[1,2]$. In the Hauser-Feshbach formalism, the cross section for this "desired" reaction takes the form:

$$
\sigma_{\alpha \chi}\left(E_{a}\right)=\sum_{J, \pi} \sigma_{\alpha}^{C N}\left(E_{e x}, J, \pi\right) G_{\chi}^{C N}\left(E_{e x}, J, \pi\right)
$$

with $\alpha$ and $\chi$ denoting the relevant entrance and exit channels, $a+A$ and $c+C$, respectively. The excitation energy $E_{e x}$ of the compound nucleus, $B^{*}$, is related to the center-of-mass energy $E_{a}$ in the entrance channel via the energy needed for separating $a$ from $B: E_{a}=E_{e x}-S_{a}(B)$. In many cases the formation cross section $\sigma(a+A \rightarrow$ $\left.B^{*}\right)=\sigma_{\alpha}^{C N}\left(E_{\alpha}\right)=\sum_{J \pi} \sigma_{\alpha}^{C N}\left(E_{e x}, J, \pi\right)$ can be calculated to a reasonable accuracy by using optical potentials, while the theoretical decay probabilities $G_{\chi}^{C N}\left(E_{e x}, J, \pi\right)$ for the different decay channels $\chi$ are often quite uncertain. The objective of the surrogate method is to determine or constrain these decay probabilities experimentally. 
In the surrogate approach, the compound nucleus $B^{*}$ is produced by means of an alternative ("surrogate"), direct reaction, $d+D \rightarrow b+B^{*}$, and the desired decay channel $\chi\left(B^{*} \rightarrow c+C\right)$ is observed in coincidence with the outgoing particle $b$. The coincidence measurement provides

$$
P_{\delta \chi}\left(E_{e x}\right)=\sum_{J, \pi} F_{\delta}^{C N}\left(E_{e x}, J, \pi\right) G_{\chi}^{C N}\left(E_{e x}, J, \pi\right),
$$

which is the probability that the $\mathrm{CN}$ was formed in the surrogate reaction with spin-parity distribution $F_{\delta}^{C N}\left(E_{e x}, J, \pi\right)$ and subsequently decayed into the channel $\chi$.

Ideally, one calculates the spin-parity distribution, $F_{\delta}^{C N}\left(E_{e x}, J, \pi\right)$, in Eq. 2 from a suitable theory that describes the formation of the compound nucleus following the direct reaction $d+D \rightarrow b+B^{*}$, measures the $P_{\delta \chi}\left(E_{e x}\right)$, and extracts the $G_{\chi}^{C N}\left(E_{e x}, J, \pi\right)$. At this time, this idealized approach has not been implemented since a combination of possible reaction mechanisms, predicted $F_{\delta}^{C N}\left(E_{e x}, J, \pi\right)$, and experimental data has not been available to unambiguously extract useful branching ratios.

More realistically, the decay of the compound nucleus is modeled in a Hauser-Feshbach-type calculation that makes use of independently available (but typically incomplete) nuclear structure information. The $G_{\chi}^{C N}\left(E_{e x}, J, \pi\right)$ obtained from such modeling are combined with calculated $F_{\delta}^{C N}\left(E_{e x}, J, \pi\right)$ to yield a prediction for $P_{\delta \chi}\left(E_{e x}\right)$. Fitting the latter to surrogate data provides further constraints on the $G_{\chi}^{C N}\left(E_{e x}, J, \pi\right)$ which can then be employed in the calculation of the desired cross section. Steps towards developing this modeling approach have been taken for measurements designed to yield $(\mathrm{n}, \mathrm{f})$ cross sections $[3,4]$.

A large majority of the surrogate applications to date has relied on invoking approximations, such as the WeisskopfEwing (WE) limit of the Hauser-Feshbach theory, which treats the $G_{\chi}^{C N}(E, J, \pi)$ as independent of $J \pi$. In that limit, the cross section expression for the desired reaction reduces to a simpler factorized form, $\sigma_{\alpha \chi}\left(E_{a}\right)=\sigma_{\alpha}^{C N}\left(E_{e x}\right)$ $\times G_{\chi}^{C N}\left(E_{e x}\right)$, where the formation cross section $\sigma_{\alpha}^{C N}\left(E_{e x}\right)$ can be calculated, and the decay probability $G_{\chi}^{C N}\left(E_{e x}\right)$ is directly obtained from the surrogate measurement, $P_{\delta \chi}\left(E_{e x}\right)=$ $G_{\chi}^{C N}\left(E_{e x}\right)$. For more details, see Refs. [2,5].

Many applications of the surrogate method have employed the WE approximation. The approach has been employed and tested by various groups, using different experimental setups: Measurements aimed at extracting (n,f) cross sections were first carried out in Los Alamos in the 1970s. More recently, the CENBG group has taken measurements in Orsay [6], and the STARS/LiBerACE collaboration has carried out experiments at Lawrence Berkeley National Laboratory. These measurements illustrate that (n,f) cross sections extracted from surrogate data are consistent with direct measurements (when these are available) and/or other surrogate measurements, and can provide useful new data, e.g. by extending the energy range for which cross section data is available. Furthermore, calculations have been carried out to test the approximation schemes employed in the analysis of surrogate fission data [2]. These calculations illustrate the level of accuracy that one can under reasonable circumstances expect from the surrogate approach. Specifically, extracted fission cross sections agree with the expected cross sections to within about 10\%; The largest deviations are found at energies below about $1 \mathrm{MeV}$, and the ratio variant of the surrogate method (see Sec. 5.) typically reduces the deviations somewhat.

\section{CHALLENGES FOR CAPTURE REACTIONS}

Capture cross sections provide specific challenges for the surrogate approach:

First, the level of precision required for the cross section is often higher than in the fission case: Recent advances in modeling the astrophysical s process have resulted in requests to determine capture cross sections within a few percent and nuclear-energy applications require cross sections to within $5-10 \% \quad[7,8]$, so the question arises whether the desired accuracy can be achieved via an indirect approach. On the other hand, cross section evaluations show large deviations from each other. Evaluated (n, $\gamma$ ) cross sections for the s-process branch point ${ }^{95} \mathrm{Zr}$, for example, differ by roughly a factor of four.

Secondly, it is the low-energy regime that is relevant to many applications. For s-process applications, for example, one needs cross sections from a few $\mathrm{keV}$ to about 200 $\mathrm{keV}$. Both calculations and measurements have shown that this is the energy range for which the Weisskopf-Ewing approximation typically breaks down. Consequently, the difference between the $J \pi$ populations of the compound nucleus produced in the surrogate and desired reactions, the so-called 'spin-parity mismatch,' needs to be accounted for.

Furthermore, detecting the exit channel of interest $(\gamma$ decay in this case) in a surrogate experiment can be difficult. Most experiments determine the relevant coincidence probability by gating on a $\gamma$ ray that is characteristic of the residual nucleus. However, the quantity of interest, the total probability for decay into the $\gamma$ channel is typically inferred through the measurement of a few specific $\gamma$ rays in the decaying residual nucleus. A model calculation or other experimental constraints have to be used to connect this quantity to the quantity of interest, namely the sum of all $\gamma$ cascades leading to the ground state of the residual nucleus. In contrast, in the fission case, it is possible to directly measure the quantity of interest, namely fission probabilities, by detecting the emitted fission fragments.

To assess the feasibility of obtaining useful capture cross section information from surrogate measurements, one has to address the above challenges. 1) Achieving an accuracy of a few percent is challenging, but constraining an unknown (n, $\gamma)$ cross section to within $20-30 \%$ could be considered a meaningful improvement of the situation. 2) Accounting for spin-parity differences between desired (neutron-induced) and surrogate reaction is not only important for capture reactions, but will also improve the reliability of the extracted fission cross sections. 3) The specific $\gamma$-cascade path that occurs in the deexcitation of 
a compound nucleus is known to depend sensitively on the spin-parity distribution of that nucleus prior to decay. This provides not only a challenge, but also an opportunity, as this sensitivity can be employed to constrain theoretical predictions of the surrogate spin-parity distributions.

\section{CASE STUDIES}

Theoretical studies have been carried out to assess the feasibility of obtaining capture cross sections from surrogate measurements and to determine promising candidates for such measurements. The strategy followed here is to extract information from Hauser-Feshbach calculations that have been adjusted to reproduce known cross sections (capture and fission). The $\gamma$-channel probabilities shown in Fig. 1 have been obtained via this procedure.

Panel a) of Fig. 1 illustrates that the branching ratios $G_{\gamma}(E, J, \pi)$ can depend very sensitively on angular momentum and parity of the decaying nucleus. In the energy regime considered, the decay of the $\mathrm{CN}{ }^{92} \mathrm{Zr}$ proceeds by $\gamma$ or neutron emission, with negligible contributions from other channels. Due to the low level density in the neighboring nucleus ${ }^{91} \mathrm{Zr}$, very few neutron decay channels are available; the opening of each new channel corresponds to a slope discontinuity in one or more $\gamma$-branching ratios. This, and the fact that the neutron decay is dominated by low partial waves ( $\mathrm{s}$ and $\mathrm{p}$ ), leads to $\gamma$-decay probabilities that are very sensitive to the $\mathrm{CN} J \pi$ population. It is clear that the WE approximation is not valid in this region.

For actinides, the behavior of the branching ratios $G_{\gamma}^{C N}(E, J, \pi)$ is governed by the competition of fission, neutron emission, and $\gamma$ decay. For ${ }^{236} \mathrm{U}$, fission competes with $\gamma$ emission below the neutron separation threshold, resulting in $G_{\gamma}^{C N}(E, J, \pi)<1$ at $E_{e x}=7.55 \mathrm{MeV}\left(E_{n}=\right.$ $0)$, as seen in panel c). While the $G_{\gamma}^{C N}(E, J, \pi)$ for $J=$ $0-6$ are very similar to each other for $E_{n}>1 \mathrm{MeV}$, they differ more significantly from each other below 1 $\mathrm{MeV}$. For energies above $E_{n} \approx 1.5 \mathrm{MeV}$, all branching ratios exhibit roughly the same energy dependence, but the $G_{\gamma}^{C N}(E, J, \pi)$ associated with the higher values $J=9,12$ differ from those for $J=0,3$ by factors ranging from 0.5 to 3 . The rough equality of the $\gamma$ probabilities for $J \leq 6$ and the significant differences in the probabilities for larger $J$ values is a consequence of the breakdown of the WE approximation due to the spin-cutoff effect in the level densities (see Ref. [5]). An increased probability for ${ }^{236} \mathrm{U}$ states with larger $J$ values to decay via $\gamma$ emission is not surprising, as s-wave neutron emission from these states is hindered at low energies due to angular-momentum selection rules, but also not immediately obvious, as the fission channel has to be considered. The situation is clearer for the rare-earth case, where neutron and $\gamma$ emission are the only significant decay modes. For actinides, we expect, based on these calculations, the cross sections obtained in the WE approximation to be limited in accuracy.

In panel b), we show $\gamma$ branching ratios $G_{\gamma}^{C N}(E, J, \pi)$ for the decay of ${ }^{156} \mathrm{Gd}$. Since the fission channel is absent, and
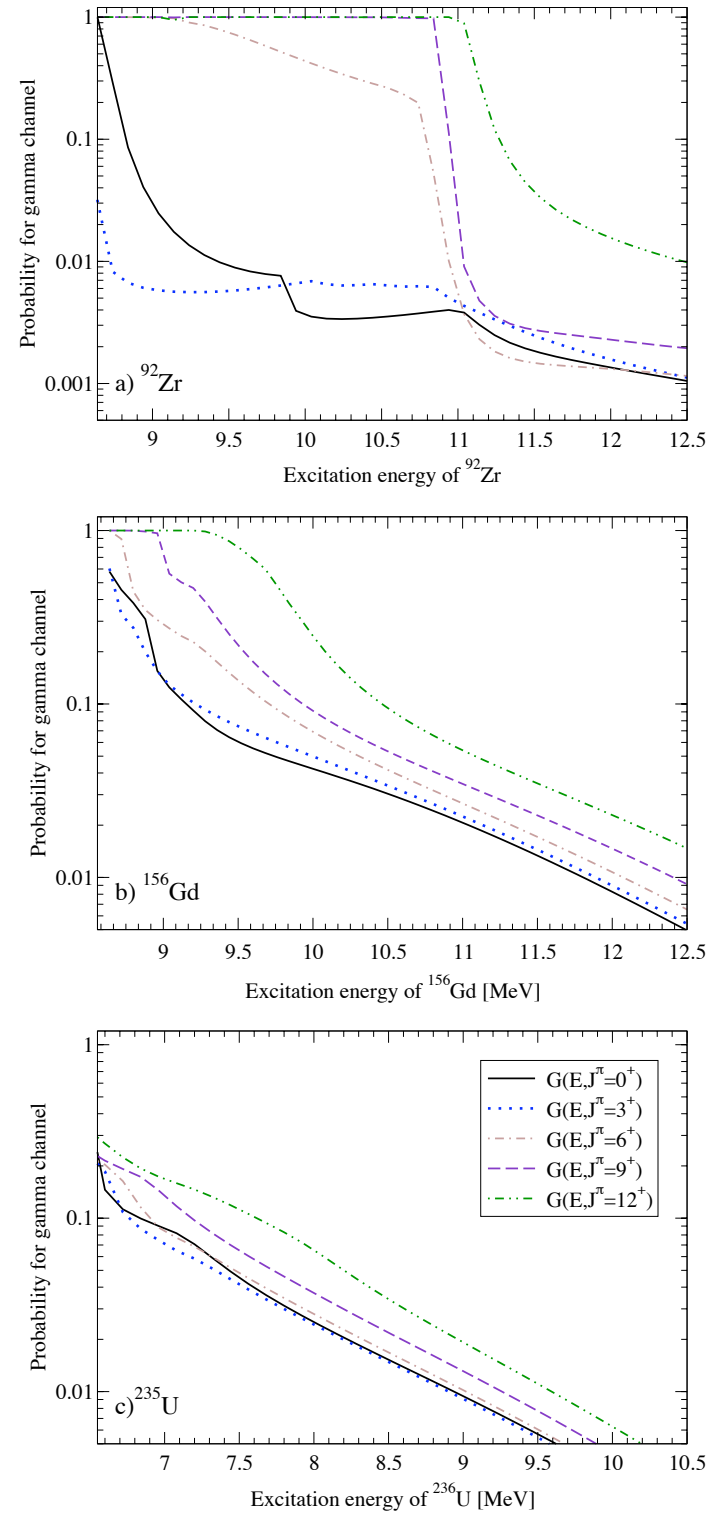

Fig. 1. Calculated $\gamma$-decay probabilities $G_{\gamma}^{C N}(E, J, \pi)$, for ${ }^{92} \mathrm{Zr}$,

${ }^{156} \mathrm{Gd}$, and ${ }^{236} \mathrm{U}$. Shown is the probability that the compound nucleus, when produced with a specific $J \pi$ combination, decays via the $\gamma$ channel. The excitation energies shown correspond to incident-neutron energies of 0-4 MeV. Only positive-parity decay probabilities are given.

cross sections for charged-particle channels are very small, all $G_{\gamma}^{C N}(E, J, \pi)$ equal one below the neutron separation energy; their behavior above $S_{n}$ is governed by the competition of $\gamma$-decay and neutron evaporation. The dependence on the spin of the decaying nucleus is stronger than in the actinide case, but significantly less than that found for the ${ }^{92} \mathrm{Zr}$ example; a parity dependence exists, but is not shown here. For energies below about $1 \mathrm{MeV}$, the branching ratios show effects of discrete levels in the neighboring nuclei; above that energy, the $G_{\gamma}^{C N}(E, J, \pi)$ have a smooth energy 
dependence. While $\gamma$-branching ratios associated with small angular-momentum values $(J \leq 3)$ are seen to drop rapidly right above the neutron separation energies, those related to larger $J$ values remain high $\left(G_{\gamma}^{C N}(E, J, \pi)=1\right)$ for several hundreds of $\mathrm{keV}$ above the neutron threshold. For these higher- $J$ states, neutron evaporation is hindered at low energies, where s-wave neutron transmission dominates, since the residual ${ }^{155} \mathrm{Gd}$ nucleus contains few high-spin states to which the decay could occur. With increasing excitation energy, states with higher spins become available in the neighboring nuclei and p-wave and d-wave transmission begin to compete - neutron evaporation becomes dominant. As the excitation energy increases above values that correspond to $E_{n} \approx 1.5 \mathrm{MeV}$, the $G_{\gamma}^{C N}(E, J, \pi)$ begin to converge slowly. However, within the energy range considered, no particular region can be identified for which the WE limit is clearly reached.

Whether it is reasonable to employ the WE approximation for a particular reaction depends not only on the energy regime considered, but also on the range of angular momenta populated in both the desired and surrogate reactions. It is possible that one reaction, for example the desired reaction, populates a narrow range of spins, while the other involves a wider range of angular-momentum values.

The effects of the spin-parity mismatch can be further explored by using schematic spin-parity distributions, $F_{\delta}^{C N}$ $(E, J, \pi)$, to simulate surrogate coincidence data via Eq. 2 . The calculated $P_{\delta \gamma}^{s i m}(E)=\sum_{J, \pi} F_{\delta}^{C N}(E, J, \pi) G_{\gamma}^{C N}(E, J, \pi)$ can be used in a WE 'analysis' to yield the desired cross section, $\sigma_{n, \gamma}^{W E, s i m}(E)=\sigma_{n}^{C N}(E) P_{\delta \gamma}^{s i m}(E)$, where $\sigma_{n}^{C N}(E)$ denotes the $\mathrm{CN}$ formation cross section. The range of cross sections, $\sigma_{n, \gamma}^{W E, s i m}(E)$, obtained by varying the simulated spin distributions within reasonable limits provides a measure of the uncertainty in the extracted cross section due to the use of the WE approximation. For the zirconium region such sensitivity analysis was carried out by Forssén et al. [9]. An order-of-magnitude difference between the known reference cross section for ${ }^{91} \mathrm{Zr}(\mathrm{n}, \gamma)$ and that extracted from the simulation was found, indicating that using the WE approximation for the this region of the nuclear chart is indeed not appropriate.

Discrepancies between extracted and reference (i.e. evaluated) cross sections are expected to be smaller for the deformed rare-earth and actinide cases, since the level densities in those regions are much higher than in the zirconium region. Recent studies [5] show that this is indeed the case. Results for the ${ }^{155} \mathrm{Gd}(\mathrm{n}, \gamma)$ and ${ }^{235} \mathrm{U}(\mathrm{n}, \gamma)$ examples are shown in Fig. 2. Plotted are the reference cross sections, obtained by fitting Hauser-Feshbach calculations to direct measurements, and four cross sections extracted from simulated surrogate data; the spin distributions are shown in Fig. 3.

The ${ }^{235} \mathrm{U}$ ground state has $J^{\pi}=7 / 2^{+}$, and the $\mathrm{CN}{ }^{236} \mathrm{U}$ produced in the neutron-induced reaction has an approximate spread in the $J^{\pi}$ distribution of $2 \leq J \leq 6$ for $E_{n} \leq$ $0.1 \mathrm{MeV}$, and $0 \leq J \leq 10$ for $E_{n} \approx 3 \mathrm{MeV}$, i.e. there is significant overlap between those spin-parity distributions and the schematic distributions 1,2, 4. The WE analysis of
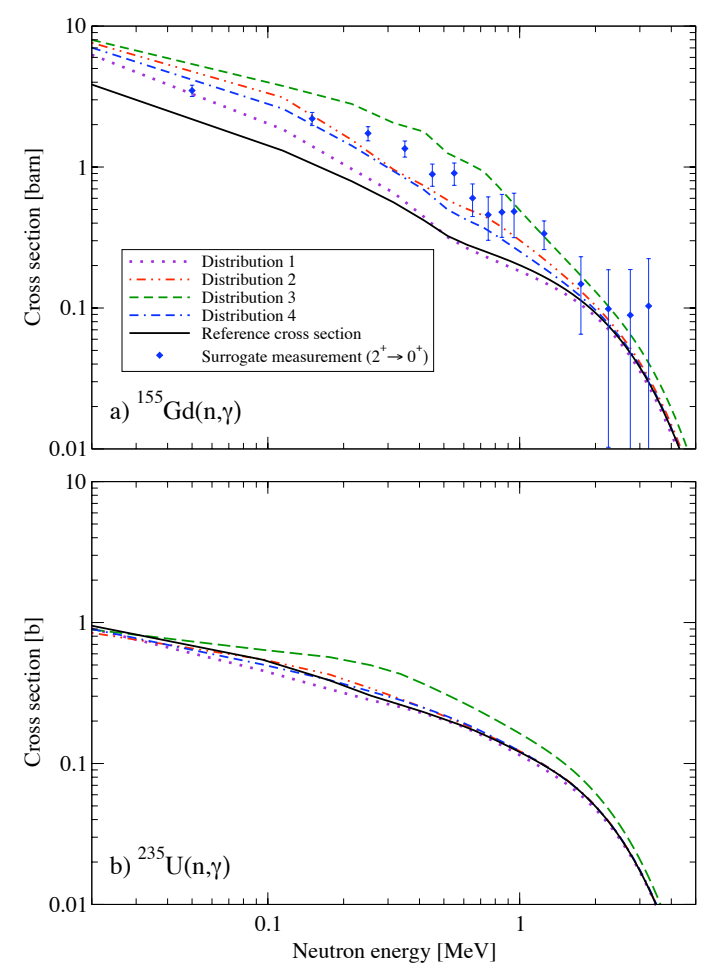

Fig. 2. Weisskopf-Ewing estimates for the a) ${ }^{155} \mathrm{Gd}(\mathrm{n}, \gamma)$ and b) ${ }^{235} \mathrm{U}(\mathrm{n}, \gamma)$ cross sections, extracted from analyses of simulated surrogate experiments, for four different compound-nuclear $J \pi$ distributions. For Gd, results from a Weisskopf-Ewing analysis of surrogate ${ }^{156} \mathrm{Gd}(\mathrm{p}, \mathrm{p}$ ') data are also shown. The reference cross sections were obtained by adjusting the parameters for the Hauser-Feshbach calculation to reproduce direct measurements.

the simulated surrogate data results indeed in cross sections that are similar to the reference cross section; in Fig. $2 b$ the curves are seen to cluster around the (n, $\gamma)$ reference result. The cross section extracted for distribution 3, however, shows sizable deviations, illustrating the limitation of the WE approximation for $(\mathrm{n}, \gamma)$ reactions in this mass region.

The spin distribution for the $\mathrm{n}+{ }^{155} \mathrm{Gd}$ case $\left({ }^{155} \mathrm{Gd}\right.$ ground state has $J^{\pi}=3 / 2^{-}$) has a spread of approximately $1 \leq J \leq 4$ for $E_{n}$ below about $1 \mathrm{MeV}$, and values between 0 and 6 for $E_{n} \approx 1.5 \mathrm{MeV}$, i.e. there is little overlap with distributions 2,3 , and 4 for the whole range of energies considered. This, and the fact that the $G_{\gamma}^{C N}(E, J, \pi)$ are more sensitive to angular momentum and parity than those relevant to the uranium case, leads to cross sections that, when obtained in the WE approximation, show poor agreement with the reference case, as can be seen in Fig. 2a).

Also shown in Fig. 2a) are results from an actual surrogate experiment, carried out by the STARS/ LIBERACE collaboration at the 88-inch cyclotron at Lawrence Berkeley National Laboratory [10]: The symbols with y error bars indicate the cross section obtained from a WE analysis of a ${ }^{156} \mathrm{Gd}\left(p, p^{\prime}\right)$ measurement with $22-\mathrm{MeV}$ protons. Protons were detected in coincidence with the $\gamma$-ray from the $2^{+} \rightarrow$ $0^{+}$transition in the ${ }^{156} \mathrm{Gd}$ ground-state band (no model- 

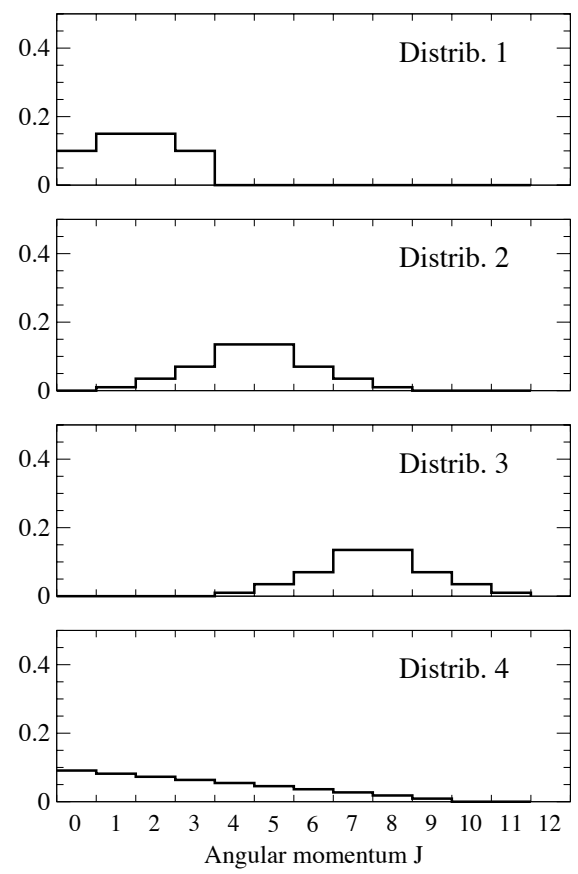

Fig. 3. Schematic spin-parity distributions, selected to simulate the compound nucleus created in the surrogate reaction.

ing was employed to account for $\gamma$-cascades that bypass these transitions). The extracted cross section falls, for the most part, between the calculated curves. It is somewhat larger than the curve corresponding to distribution 2, which indicates that the $\left(p, p^{\prime}\right)$ reaction produced a spin-parity distribution which contained $J$-values above 5-6. The cross section extracted from the surrogate measurement is a factor of 2-3 larger than the reference cross section. Clearly, it is important to correct for the spin-parity mismatch.

\section{IMPROVING THE CROSS SECTION RESULTS}

Several strategies can be considered in order to improve the reliability and accuracy of neutron-capture cross sections extracted from surrogate experiments.

\subsection{Ratios of coincidence probabilities}

The Surrogate Ratio approach $[2,11,12]$ is an approximation that makes use of the surrogate idea and requires the (approximate) validity of the Weisskopf-Ewing limit. In this approach, the ratio $R(E)=\sigma_{\alpha_{1} \chi_{1}} / \sigma_{\alpha_{2} \chi_{2}}$ of the cross sections of two $\mathrm{CN}$ reactions is measured, using two surrogate experiments. An independent determination of the cross section $\sigma_{\alpha_{2} \chi_{2}}$ can then be used to deduce $\sigma_{\alpha_{1} \chi_{1}}$. An advantage of using the ratio method is the fact that it eliminates the need to accurately measure the total number of surrogate reaction events $\left(N_{\delta}\right)$, since one determines the ratio of coincidence probabilities $P_{\delta_{1} \chi_{1}} / P_{\delta_{2} \chi_{2}}$, rather than an absolute probability $P_{\delta \chi}=N_{\delta \chi} / N_{\delta}$. Furthermore, there are indications that small to moderate deviations from the WE assumption might cancel in this approach [2]. Cross sections for $(\mathrm{n}, \mathrm{f})$ reactions extracted in the ratio approximation have been tested for consistency with results from direct measurements, complementary surrogate experiments $[12$, 13], and theoretical simulations [2]. For (n,f) reactions one typically finds that the spin-parity mismatch between the desired and surrogate reactions has a much smaller effect on the extracted cross section than in an approach that uses the WE approximation and absolute probabilities. Also, deviations caused by pre-equilibrium effects are diminished, thus improving the overall agreement between extracted and expected cross sections.

For $(\mathrm{n}, \gamma)$ reactions, the situation is more subtle: In situations in which the WE approximation yields a cross section that is in reasonable agreement with a directlymeasured result, the ratio method can yield further improvements. For the uranium region, this has been shown in Ref. [5]. A study of the gadolinium region, however, indicates that the WE approximation overestimates the (n, $\gamma)$ cross section by factors which depend more strongly on the nucleus under consideration $[5,10]$. For the ${ }^{155} \mathrm{Gd}(\mathrm{n}, \gamma)$ and ${ }^{157} \mathrm{Gd}(\mathrm{n}, \gamma)$ cross sections, the differences were large, despite the structural similarities of the relevant nuclei. The ratio approach resulted in ${ }^{155} \mathrm{Gd}(\mathrm{n}, \gamma)$ cross sections that were, for the schematic spin distributions considered here, too large by as much as $250 \%$ for energies below about $E_{n}=$ $0.7 \mathrm{MeV}$; at the same time, they did converge more rapidly to the correct result than the cross sections obtained from absolute probabilities. Overall, the ratio approach was found to reduce, but not eliminate, the effect of the spin-parity mismatch on the extracted cross sections for energies where the WE assumption is a poor approximation.

\subsection{Normalizing calculated cross sections}

One might try to utilize the fact that surrogate experiments can, in principle, provide decay data for a wide range of energies, including energies for which the WE limit is approximately valid. This approach was investigated for zirconium [9]. As long as $\mathrm{CN}$ angular momenta below about 6 are involved, the WE approximation was found to be roughly valid for $E_{n} \approx 3 \mathrm{MeV}$ (see Fig.1a), making a fit to surrogate decay data in this region significantly less sensitive to the predicted $J \pi$ population than at lower energies. In addition, the sensitivity studies presented in Ref. [9] showed that modeling errors in the s-wave average radiative width $\left\langle\Gamma_{\gamma}\right\rangle_{0}$, or in the level spacing $D_{0}$, affect primarily the magnitude of the calculated cross section, but do not change the energy dependence of the cross section much. It was shown that several different decay models, representing typical uncertainties present in cross section evaluations, could be normalized to surrogate data in this energy region, despite incomplete information on surrogate spin-parity populations. The cross sections extracted using this procedure were found to be in remarkable agreement with results from an evaluation. The reason for the success of this approach was attributed to the linear relationship between variations of the level density formula, or $\gamma$ - 
strength function, and the corresponding effect on the $\gamma$ decay branching ratios. This normalization approach is worth considering when it is possible to collect surrogate data with sufficient statistics in the relevant energy region.

\subsection{Minimizing the effects of the spin-parity match}

A primary challenge for the surrogate approach lies in accounting for the spin-parity mismatch between the desired and surrogate reactions. When it is possible to identify a surrogate reaction (i.e. a reaction mechanism, projectiletarget combination, beam energy, outgoing-particle angle) that approximately reproduces the spin-parity distribution of the desired reaction,

$$
F_{\delta}^{C N}\left(E_{e x}, J, \pi\right) \approx F_{\alpha}^{C N}\left(E_{e x}, J, \pi\right) \equiv \frac{\sigma_{\alpha}^{C N}\left(E_{e x}, J, \pi\right)}{\sum_{J^{\prime}, \pi^{\prime}} \sigma_{\alpha}^{C N}\left(E_{e x}, J^{\prime}, \pi^{\prime}\right)},(3)
$$

where $F_{\alpha}^{C N}$ is the compound-nuclear $J \pi$ population in the desired reaction, the situation simplifes greatly, as in this limit, we find $\sigma_{\alpha \chi}\left(E_{a}\right) \approx \sigma_{\alpha}^{C N}\left(E_{e x}\right) \times P_{\delta \chi}^{e x p}\left(E_{e x}\right)$, where the cross section for forming the compound nucleus at energy $E_{e x}, \sigma_{\alpha}^{C N}\left(E_{e x}\right) \equiv \sum_{J \pi} \sigma_{\alpha}^{C N}\left(E_{e x}, J, \pi\right)$, is calculated using a suitable optical potential, and $P_{\delta \chi}^{\text {exp }}\left(E_{\text {ex }}\right)$ is determined from the experiment. While it is sometimes argued that a given surrogate experiment approximately satisfies Eq. 3, there has not been sufficient evidence to support such claims.

\subsection{Predicting CN spin-parity distributions}

Predicting the spin-parity distribution for a $\mathrm{CN}$ produced in a surrogate reaction requires a careful consideration of the reaction mechanisms involved. It involves a description of direct reactions that populate highly-excited, unbound states, and the damping of these doorway states into more complicated configurations that lead to the $\mathrm{CN}$. The possibility that the intermediate system produced in a surrogate reaction does not lead to the $\mathrm{CN}$ of interest, but decays via non-equilibrium particle emission prior to reaching the compound stage, has to be considered. The probability for this process needs to be calculated, along with its dependence and influence on angular momentum, parity, and energy of the decaying nuclear system [14]. Developing a reliable theoretical description of the formation of a $\mathrm{CN}$ following a direct reaction will be crucially important for improving the accuracy and reliability of the surrogate method.

Efforts to predict the spin-parity distributions need to be accompanied by the development of experimental methods that can test the predictions. While the strong spin-parity dependence of the observables used to tag the exit channel makes extracting $(\mathrm{n}, \gamma)$ cross sections from surrogate measurements very challenging, it also provides valuable information. In particular, simultaneously measuring the yields of several $\gamma$-ray transitions of a decaying compound nucleus can provide signatures for the spin-parity distribution of the CN prior to decay. Relative $\gamma$-ray yields for the decay of even-even gadolinium nuclei have recently been measured [10] and methods are being developed to use this information in order to improve the (n, $\gamma)$ cross sections determined from surrogate experiments.

\section{CONCLUDING REMARKS}

Successful surrogate measurements of $(n, f)$ cross sections have been carried out by several groups, using different experimental setups and various projectile-target combinations and direct-reaction mechanisms. Almost all analyses of the data have employed the Weisskopf-Ewing approximation, which is not expected to be valid at low energies $\left(E_{n}<1-2 \mathrm{MeV}\right)$. Cross section measurements for $(\mathrm{n}, \gamma)$ reactions provide new challenges, due to the precision required, the low neutron energies involved, and the necessity to identify the $\gamma$ decay channel. Case studies help to assess the feasibility and limitations of surrogate measurements of $(\mathrm{n}, \gamma)$ cross sections; the Weisskopf-Ewing approximation is found to yield unsatisfactory results. Approaches for circumventing specific difficulties and for improving the accuracy and reliability of neutron-capture cross sections extracted from surrogate experiments are presently being developed.

\section{ACKNOWLEDGMENTS}

This work was performed under the auspices of the U.S. Department of Energy by Lawrence Livermore National Laboratory under contract DE-AC52-07NA27344.

\section{REFERENCES}

[1] J. D. Cramer and H. C. Britt Nucl. Sci. and Eng., 41, 177 (1970); H. C. Britt and J. B. Wilhelmy Nucl. Sci. and Eng., 72, 222 (1979).

[2] J. E. Escher and F. S. Dietrich, Phys. Rev. C, 74054601 (2006).

[3] B. L. Andersen, B. B. Back, and J. M. Bang, Nucl. Phys. A, 147, 33 (1970); B. B. Back, O. Hansen, H. C. Britt, and J. D. Garrett, Phys. Rev. C, 91924 (1974).

[4] W. Younes and H. C. Britt Phys. Rev. C, 67, 024610 (2003).

[5] J. E. Escher and F. S. Dietrich, Phys. Rev. C, 81, 024612 (2010).

[6] B. Jurado et al., in International Conference on Nuclear Data for Science and Technology (ND2007), Nice, France, April 22-27, 2007, p. 331, EDP Sciences, 2008.

[7] F. Kappeler and A. Mengoni, Nuclear Physics A, 777, 291 (2006).

[8] G. Aliberti, G. Palmiotti, M. Salvatores, et al. Annals of Nuclear Energy, 33, 700 (2006).

[9] C. Forssén, F. Dietrich, J. Escher, R. Hoffman, and K. Kelley, Phys. Rev. C, 75, 055807 (2007).

[10] N. D. Scielzo, J. E. Escher, J. M. Allmond, et al., Phys. Rev. $C, \mathbf{8 1}, 034608$ (2010).

[11] C. Plettner et al., Phys. Rev. C, 71, 051602(R) (2005).

[12] J. Burke, L. Bernstein, J. Escher, et al., Phys. Rev. C, 73, 054604 (2006).

[13] S. R. Lesher, J. T. Burke, L. A. Bernstein, et al., Physical Review C, 79, 044609 (2009).

[14] F. S. Dietrich, AIP Conference Proceedings, 1005, 125 (2008). 